\title{
Large-Scale Spreading of Deep Waters in the Western Mediterranean Sea by Submesoscale Coherent Eddies
}

\author{
P. TESTOR AND J.-C. GASCARD \\ Laboratoire d'Océanographie Dynamique et de Climatologie, Université Pierre et Marie Curie, Paris, France
}

(Manuscript received 15 June 2001, in final form 6 June 2002)

ABSTRACT

\begin{abstract}
Two large-scale free-drifting isobaric-floats experiments, "SOFARGOS"/Marine Science and Technology Programme, phase 2 (MAST2) and Mass Transfer and Ecosystem Response (MATER)/MAST3, undertaken in 1994-95 in the northwestern Mediterranean Sea and in 1997-98 in the Algerian Basin, respectively, have revealed for the first time that Western Mediterranean Deep Water, newly formed by deep convection in the Gulf of Lion (the so-called Medoc site), can be advected several hundreds of kilometers away from the formation area by anticyclonic submesoscale coherent vortices (SCVs). This behavior implies that SCVs participate actively in the large-scale thermohaline circulation and deep ventilation of the western Mediterranean Sea. These SCVs are characterized by small radius $(\sim 5 \mathrm{~km})$, very low potential vorticity, high aspect ratio $(\sim 0.1)$, and extended lifetime $(>0.5 \mathrm{yr})$.
\end{abstract}

\section{Introduction}

The Greenland Sea, the Labrador Sea, and the Mediterranean Sea are the few particular seas exposed to very strong atmospheric conditions in winter in which localized open-ocean regions characterized by weak stratification allow vertical mixing to reach very great depths. The main mechanism involved in the process is so-called deep convection. This phenomenon occurs in particular in the Gulf of Lion (northwestern Mediterranean Sea) and has been studied thoroughly since the 1970s [MEDOC Group (1970); see Killworth (1983) and Marshall and Schott (1999) for a review]. Still, many aspects of open-ocean deep convection remain unclear, especially those concerning the large-scale spreading of the newly-formed deep waters.

Based on temperature time series from a current meter moored on the continental slope of the Gulf of Lion, Send et al. (1996) indicated that a significant part of the newly formed deep water can escape from the mixed patch into the boundary current (the so-called LiguroProvençal or Northern Current) running along the continental slope of the Gulf of Lion. Numerical simulations of Madec et al. (1991) also suggested a "bleeding" of the newly formed Mediterranean deep waters from the mixed patch into the northern boundary current. Other simulations (Legg and Marshall 1993; Legg et al. 1996, 1998; Jones and Marshall 1997) suggested that lateral

Corresponding author address: P. Testor, Laboratoire d'Océanographie Dynamique et de Climatologie, Université P. et M. Curie, 4 place Jussieu, Paris 75252, France.

E-mail: testor@lodyc.jussieu.fr fluxes by geostrophic eddies generated by baroclinic instability around the mixed patch must play a major role in contributing to the spreading of the newly formed waters out of the patch. Laboratory experiments performed by Maxworthy and Narimousa (1994) also apparently confirm the dominant role of eddies in the spreading of the newly formed homogeneous waters. The role of eddies in the lateral exchanges between the mixed patch and the stratified environment has not yet been quantified for long timescales and large spatial scales by either direct observations or laboratory experiments. Because numerical studies are limited in time and space, they do not describe the long-term fate of newly formed deep waters either.

The "SOFARGOS" Lagrangian floats experiment undertaken in the northwestern Mediterranean Sea in 1994-95 brought evidence of newly formed deep waters being advected several hundreds of kilometers away from the convection site by anticyclonic submesoscale coherent vortices (SCVs). These eddies of 5-km radius are similar to the Local Dynamics Experiment SCV presented by McWilliams (1985) and involved in the transport of newly formed Labrador Sea deep waters in the middle of the Atlantic Ocean. In the Mediterranean Sea, floats were observed to circle SCVs for periods of 5 months. Although the generation mechanism is still uncertain at this point, SCVs may be related to baroclinic instability generated in the deep convection area. This first experiment was limited in time and space and did not show how far SCVs could propagate ultimately or how long they could live. The Mass Transfer and Ecosystem Response (MATER) experiment in the south- 
southwestern Mediterranean Basin in 1997-98 also revealed the existence of SCVs similar to those observed in the northern basin during SOFARGOS and tending to indicate a similar origin, longer lifetime, and larger spreading. We present here these new results regarding large-scale spreading of newly formed Western Mediterranean Deep Waters by submesoscale anticyclonic coherent vortices.

\section{Lagrangian experiments}

RAFOS floats were used intensively in 1994-95 and 1997-98 in the north and southwestern Mediterranean Sea, respectively, during two experiments (SOFARGOS 1994-95 and MATER 1997-98) funded by the European Economic Community (EEC) under the Marine Science and Technology Programme, phase 2 (MAST2) and MAST3 [third and fourth EEC Framework Programs, MAST2 (1996) and MAST3 (1999)]. These floats are quasi-isobaric because of their compressibility, which is much lower than that of seawater. They are advected by horizontal currents at a predetermined and quasi-constant depth. They are tracked six times per day (typically over a year) by acoustic triangulation using an array of moored low-frequency sound sources $(780 \mathrm{~Hz})$ transmitting long-range propagating acoustic signals. The floats are tracked every $4 \mathrm{~h}$ by recording (with a precision of $0.1 \mathrm{~s}$ ) the arrival time of signals transmitted by a minimum of three acoustic sources. The floats also measure in situ temperature and in situ pressure. At the end of their missions, the floats surface after dropping a dead weight and transmit data to $\mathrm{Na}-$ tional Oceanic and Atmospheric Administration (NOAA) satellites equipped with the Argos data transmission system, operated by Service Argos, Inc., and North American CLS, Inc.

\section{The northern basin}

During the SOFARGOS experiment, 25 RAFOS floats were launched at depths varying from 350 to 1450 $\mathrm{m}$ during the winter of $1994 / 95$ to document the circulation of intermediate and deep waters in the Gulf of Lion. Ten floats were launched in December of 1994 in the Liguro-Provençal Current south of Toulon, and 15 floats were launched in January of 1995 in the deepwater formation zone (the so-called Medoc area centered around $42^{\circ} \mathrm{N}, 5^{\circ} \mathrm{E}$ ). These floats drifted underwater until July of 1995 for 7 and 5 months, respectively. Deep convection occurred early during winter 1994/95 (December-January) and reached 2000-m depth in a mixed patch stretched above the Rhône's deep-sea fan and at the crossroads of the Alps' and Pyrenées' katabatic winds (mistral and tramontane). Deep convection resulted in new Western Mediterranean Deep Water (nWMDW) characterized by a potential temperature of approximately $12.9^{\circ} \mathrm{C}$ and a salinity of approximately 38.48 psu from the surface down to $1500-\mathrm{m}$ depth and

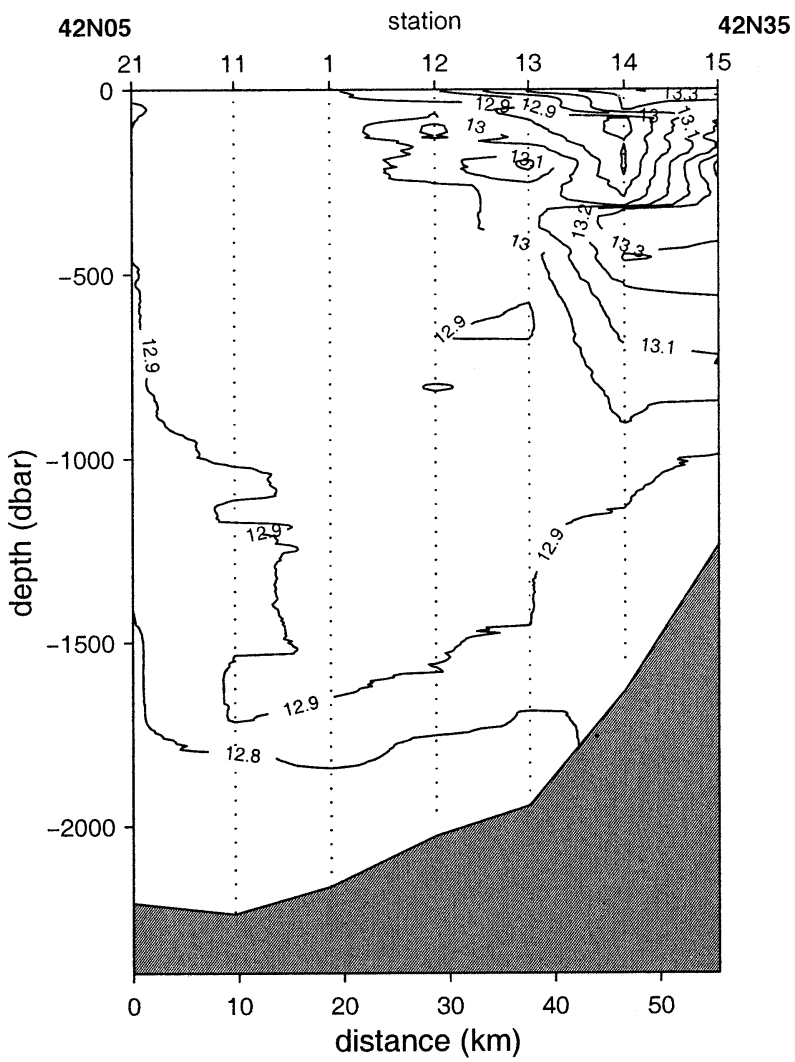

FIG. 1. Meridional section of potential temperature taken in late Jan 1995 along $4^{\circ} 30^{\prime} \mathrm{E}$ in the Gulf of Lion.

more (Fig. 1). The isobaric floats highlighted a new aspect of large-scale spreading following deep convection in this region-the role of SCVs.

\section{a. SCV-N1}

One of the most striking observations was from a float (float 93 drifting at 1400-m depth), trapped for more than 5 months inside a long-lived anticyclonic SCV (hereinafter named SCV-N1) originating from the convection area and advecting nWMDW $150 \mathrm{~km}$ away from the convection zone (Fig. 2). The float accompanied the vortex for about 5 months until it was scheduled to surface to start transmitting data. Thus, this vortex lasted for at least 5 months. During this period, the float indicated a remarkably constant potential temperature of $12.89^{\circ} \mathrm{C}$ (Fig. 3), typical of nWMDW for this particular depth, whereas old Western Mediterranean Deep Water (WMDW) is characterized by a colder potential temperature of approximately $12.8^{\circ} \mathrm{C}$. This eddy has no surface signature according to NOAA Advanced Very High Resolution Radiometer (AVHRR) SST images (within 1-km space resolution for a given accuracy of $\sim 0.1^{\circ} \mathrm{C}$ ). During a short period of time in February of 1995, another float (float 99) drifting at 350-m depth within SCV-N1 (along with float 93 at 1400-m depth) indicated that the eddy extended at least $1100 \mathrm{~m}$ ver- 


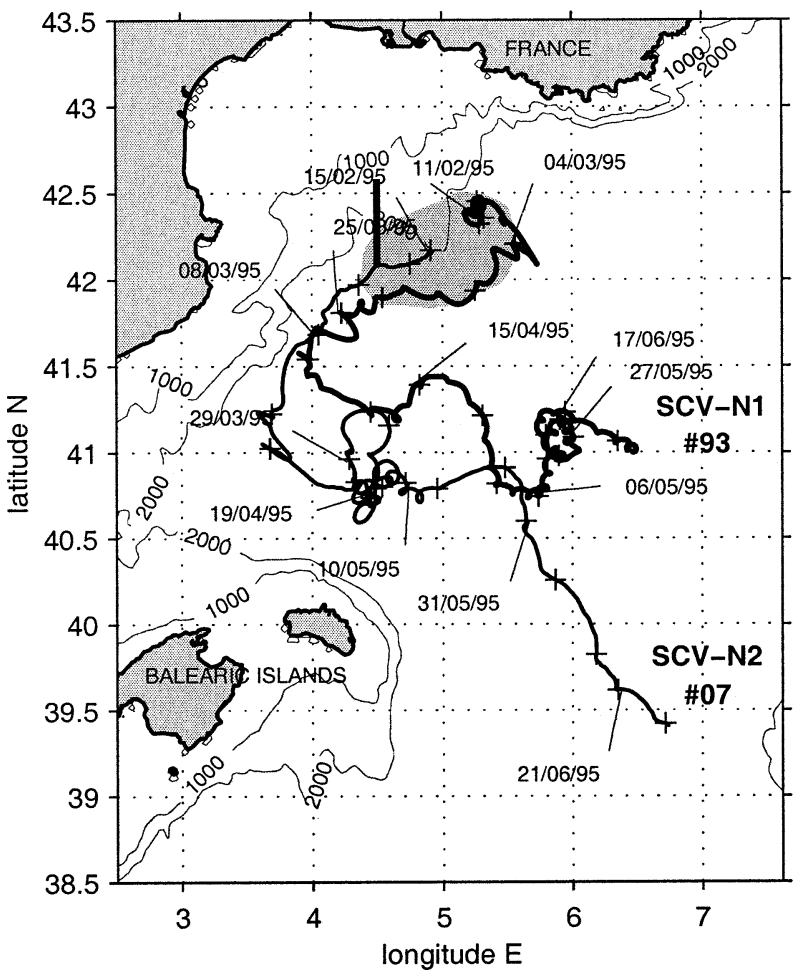

FIG. 2. Trajectories of floats 93 and 07 labeled in day/month/year (last 2 digits) revealing SCVs involved in the spreading phase of deep convection. The bold line indicates the location of the section presented in Fig. 1. The shaded area indicates the approximate mixed patch location. tically at the beginning of its life. The float at $350-\mathrm{m}$ depth measured slightly warmer temperatures (around $\left.13^{\circ}-13.05^{\circ} \mathrm{C}\right)$ than the other float $\left(12.9^{\circ} \mathrm{C}\right)$, indicating that the vortex core stratification was weak and similar to homogeneous profiles observed in the convection area in January of 1995. SCV-N1 looks like an anticyclonic subsurface eddy of very large vertical extension and aspect ratio.

An estimation of the vortex center location, by applying low-pass filtering to float fixes, allowed us to deduce an approximate radial distribution of orbital velocity (Fig. 4). Approximating this structure to be axisymmetric, one can estimate basic parameters such as relative vorticity and vortex radius, defined as the distance at which the maximum orbital velocity is observed. In Fig. 4 (top), a 2D histogram of orbital velocity illustrates the radial structure of the vortex. Orbital velocities increase with distance from the vortex center within the first $5 \mathrm{~km}$ and decrease farther away. The radial structure could be approximated to a quasi-solid body rotation near the center. The relative vorticity for an axisymmetric distribution of orbital velocity $u$ is $\zeta$ $=(1 / r) \partial(r u) / \partial r$, where $r$ is the radial distance from the center. In the case of solid-body rotation, in the core of the vortex $\zeta=(2 U) / R=4 \pi / T_{r}$ (2 times the angular velocity), where $U$ is the orbital velocity, $R$ is the corresponding radius, and $T_{r}$ is the rotational period. The vorticity of SCV-N1 is so estimated to be about $-f / 2$ ( $f$ being the Coriolis parameter) in the core of the vortex. A least squares fit to points near the center (closest to the center $<4 \mathrm{~km}$ ) leads to the same estimate.

Another float (not shown), drifting near float 93 and
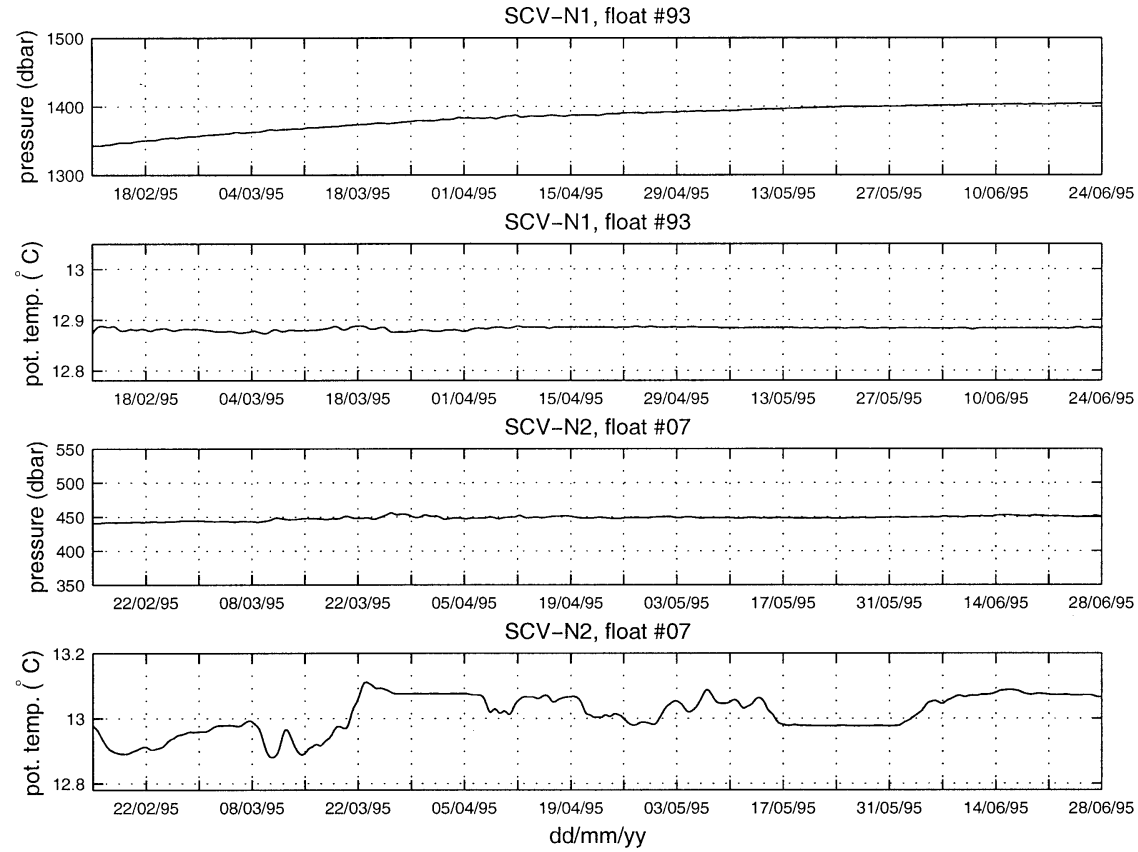

FIG. 3. Time series of potential temperature and pressure measured in situ by floats 93 and 07 drifting within SCV-N1 and SCV-N2, respectively. 

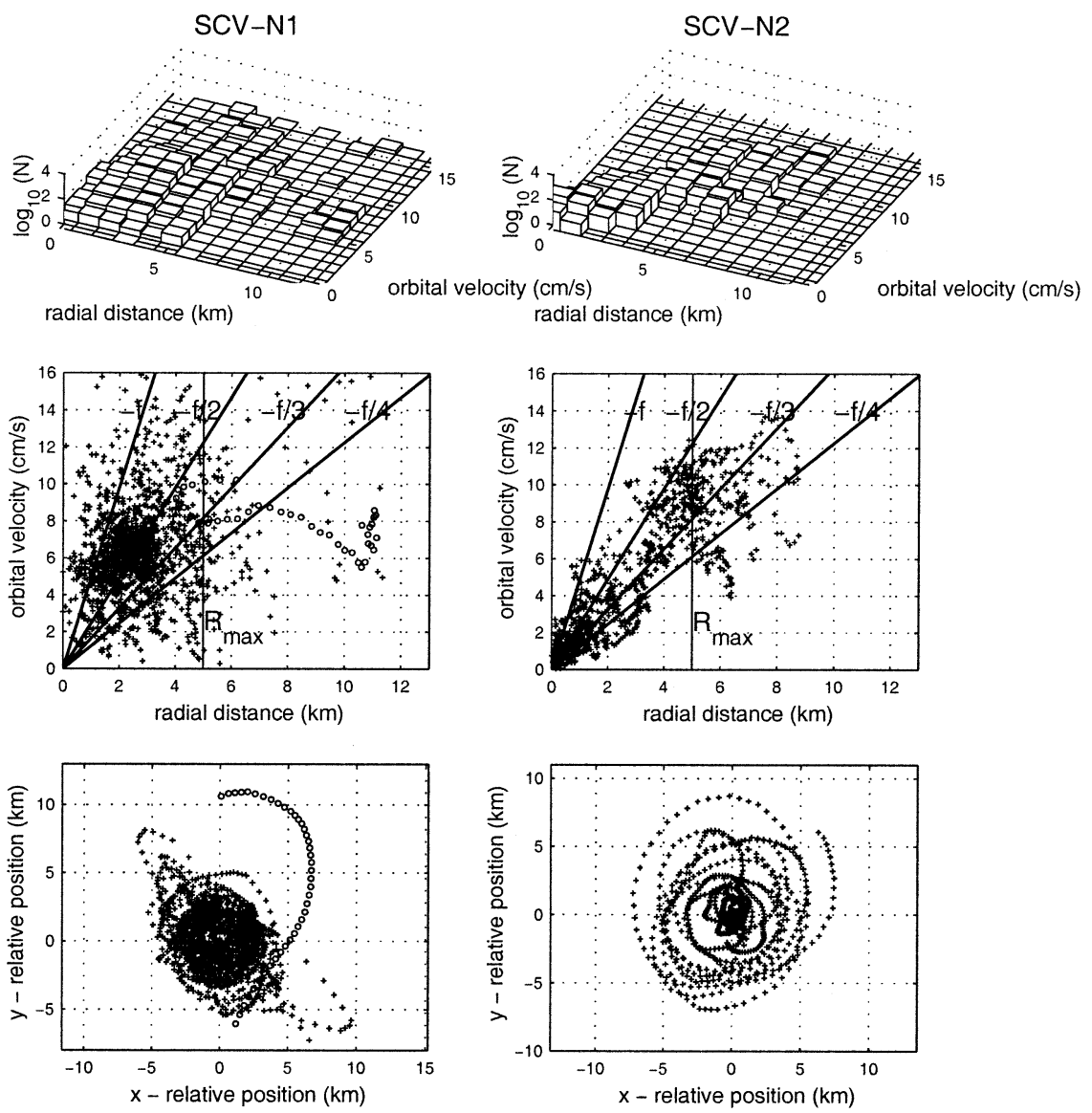

FIG. 4. (bottom) Positions of floats $93(+)$ and $07(+)$ relative to the center of SCV-N1 and SCV-N2, respectively. Float 99, at 350-m depth and trapped in SCV-N1, is also represented $(\bigcirc)$. (middle) Radial structure of the orbital velocities of SCV-N1 and SCV-N2 inferred from the estimate of the eddy center of rotation as a function of time. (top) Two-dimensional histograms of the number of observations, where $N$ is the number of measurements in each 2D class.

at about the same depth, was not influenced by the anticyclonic rotation, giving an upper bound of less than $10 \mathrm{~km}$ for horizontal eddy scale. The vortex apparently is composed of a homogeneous core extending over a 5 -km radius (distance of maximum orbital velocity) with a rotational period of approximately 3 days. This eddy radius is lower than the internal radius of deformation, which is about $15 \mathrm{~km}$ except within the convection area where it is about $5 \mathrm{~km}$ instead. The relative vorticity of $-f / 2$ implies a Rossby number Ro $=\zeta / f=-0.5$. The Burger number $\mathrm{Bu}=\left[(N H) /\left(f R_{\max }\right)\right]^{2}$ is estimated to be about 0.06 using $R_{\max }$ of about $5 \mathrm{~km}, N$ of about $2 f(N$ is the Brunt-Väisälä frequency estimated from potential density differences between depths of 500 and $1500 \mathrm{~m}$ from CTD profiles obtained from late January of 1995 in the convection area), and $H$ of about $0.6 \mathrm{~km}$, the half thickness of the vortex. This vortex fits well into the SCV definition of McWilliams (1985), because it is characterized by a strong potential vorticity anomaly, mainly from a high relative anticyclonic vorticity and low stratification, long lifetime $(>0.5 \mathrm{yr})$, and small radius as compared with the internal radius of deformation (except within the convection area).

\section{b. $S C V-N 2$}

During the same experiment, another float (float 07), drifting at 450-m depth and launched initially in the Liguro-Provençal Current (at $42^{\circ} 50^{\prime} \mathrm{N}, 5^{\circ} 35^{\prime} \mathrm{E}$ ) in December of 1994, passed through the Medoc area in February. At this time, it exhibited patterns similar to those of float 93 (Fig. 2), with anticyclonic loops with rotational periods of about 3 days from 15 February 1995 until July of 1995 (this vortex is hereinafter named SCVN2). Again, there was no signature on NOAA AVHRR SST images indicating the presence of a vortex. The radial structure of the orbital velocity inferred from an estimate of the vortex center location as a function of time (Fig. 4) leads to an associated Rossby number very similar to that of SCV-N1. The relative vorticity is estimated to be $-f / 2.4$ very near the center $(<2 \mathrm{~km})$. No relative maximum in the orbital velocity structure could 


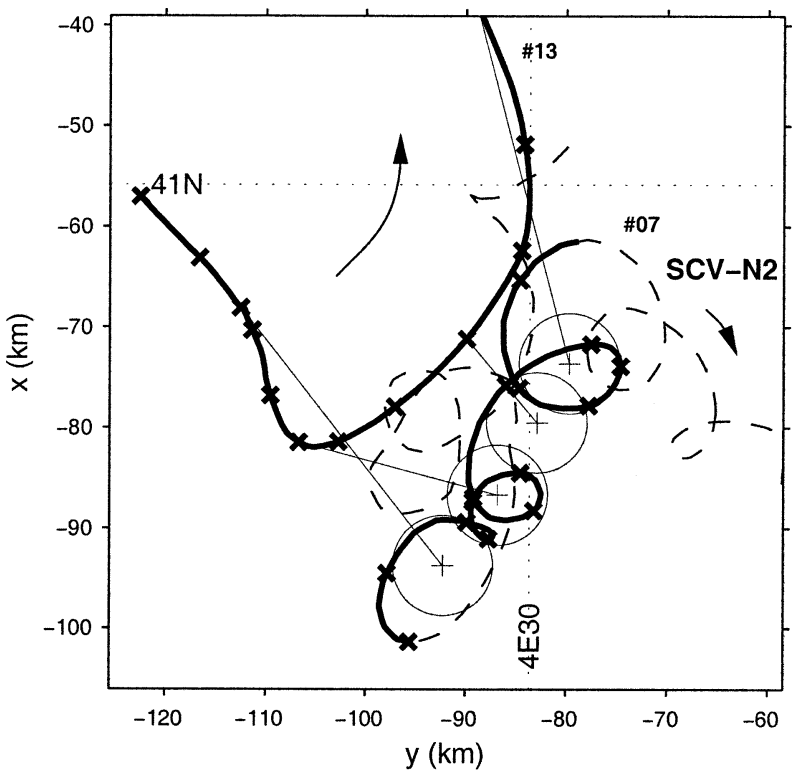

FIG. 5. Trajectory of float 07 inside SCV-N2 (dashed) and trajectories of floats 13 (situated at around 410-m depth) and float 07 (situated at around 450-m depth) (dark solid) between 22 Apr 1995 and 6 May 1995. A cross represents each day. Every 3 days, a thin circle with $5-\mathrm{km}$ radius centered on SCV-N2 center positions and a thin line between the center and float 13 position are plotted. The minimum distance between the center of SCV-N2 and float 13, which is not influenced by anticyclonic rotation, is about $10 \mathrm{~km}$.

be found. Information from another float passing nearby (about $10 \mathrm{~km}$ from the center) and uninfluenced by the vortex anticyclonic rotations gives an upper bound for the eddy radius (Fig. 5), which was thus estimated to be about $5 \mathrm{~km}$.

Float 07 first revealed temperatures $\left(12.9^{\circ}-12.97^{\circ} \mathrm{C}\right)$ typical of nWMDW at 450-m depth. Then, in late March, float 07 measured temperatures that were slightly warmer but still abnormally low, $12.97^{\circ}-13.1^{\circ} \mathrm{C}$ (Fig. $3)$, where potential temperatures of $13.2^{\circ}-13.4^{\circ} \mathrm{C}$ are more typical of the Levantine Intermediate Water (LIW) in this region. This temperature indicates nWMDW (reference value of $12.9^{\circ} \mathrm{C}$ ) already starting to warm because of the surrounding warm LIW at this level. This is confirmed by the temperature variability, which indicates that float 07 evolved in a part of this coherent vortex, subjected to temperature modifications from LIW influence.

In summary, float 07 , like float 93 , indicated a second SCV affecting the large-scale spreading of nWMDW. An interesting point is that this second SCV identified by float 07 entered the Algerian Basin $(300 \mathrm{~km}$ from the convection area) in July of 1995 . The duration of the SOFARGOS experiment (5-7 months) did not allow us to follow these two SCVs beyond that period. It is clear, however, that SCV-N1 and SCV-N2 are long-lived features capable of transporting nWMDW far from the source region.

\section{The southern basin}

In a second Lagrangian experiment (MATER), 14 floats were launched in the intermediate waters of the Algerian Basin in July of 1997 and drifted freely at about 500-700-m depth for one year. They documented the subsurface general circulation of this basin and complemented other observations taken during the Eddies and "Leddies" Interdisciplinary Study off Algeria (ELISA) operation (MAST3 1999). Two years after the SOFARGOS program, MATER floats highlighted the presence of submesoscale coherent vortices and also of a mesoscale LIW eddy in the Algerian Basin.

\section{a. LIW eddy}

Figure 6 (panel I) shows two floats (39 and 14) launched south of Sardinia in the LIW layer and trapped soon after deployment in a mesoscale eddy (marked with an L). Unlike the large anticyclonic eddies (Algerian eddies) frequently observed in the Algerian Basin (Millot 1999), the eddy has no surface signature according to NOAA AVHRR SST images. The two floats recorded the high temperatures $\left(\sim 13.6^{\circ} \mathrm{C}\right.$ at $600-\mathrm{m}$ depth and $\sim 13.4^{\circ} \mathrm{C}$ at $700-\mathrm{m}$ depth) typical of LIW observed off the coast of Sardinia (Fig. 7) and coming directly from the Tyrrhenian Sea. We have no direct indication of the vertical extension of this LIW eddy from floats, although CTD profiles taken in the area tend to indicate temperature-salinity anomalies typical of LIW between depths of 200 and $800 \mathrm{~m}$. Floats 14 and 39 were trapped initially in this LIW eddy feature until 26 December 1997. At this time, two very distinctive centers of rotation appeared [Fig. 6 (panel II)]. The center of rotation (float 14) still characterized the LIW eddy while another center of rotation (float 39) appeared belonging to another eddy (marked S1) of much smaller size and much higher rate of rotation. Float 14 remained in the LIW eddy, as indicated by its trajectory, following anticyclonic loops with rotational periods of about 3-4 weeks until April of 1998 [Fig. 6 (panels II and III)], indicating that the lifetime of this LIW eddy was greater than 5 months.

The positions of the floats relative to the estimated center of rotation of the LIW eddy are plotted in Fig. 8 along with the corresponding orbital velocities. A radial distribution of orbital velocities (Fig. 9) and an upper bound on the eddy radius from floats passing nearby (not shown) but uninfluenced by the vortex rotations can be estimated, giving a typical LIW eddy radius length scale of about $25-30 \mathrm{~km}$, a relative vorticity $\zeta$ of approximately $-f / 16$, and a Rossby number of -0.06 , very different from SCVs (Table 1) and more typical of a mesoscale eddy feature. Indeed, the eddy's radius is about 2 times the deformation radius $(\sim 15-$ $20 \mathrm{~km}$ ) for this area.

\section{b. SCV-S1 and SCV-S2}

Float 39 left the LIW eddy on 26 December 1997 (section 4a and Fig. 6) and began to drift without any 
(I)

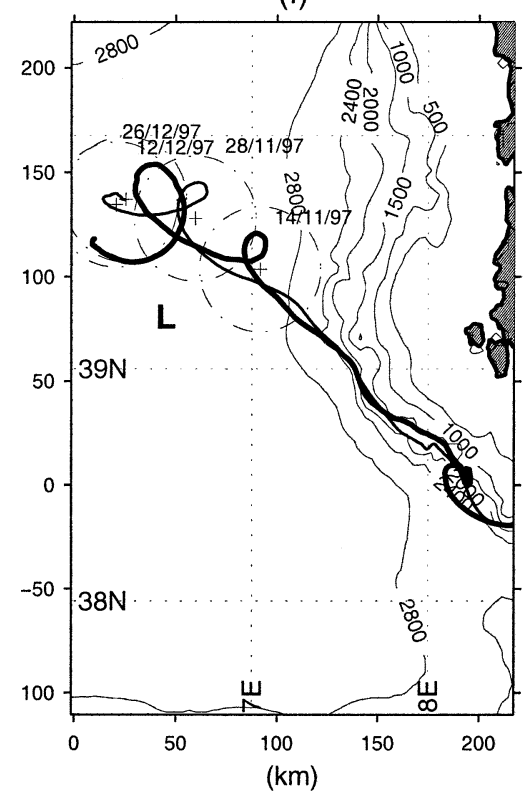

(II)

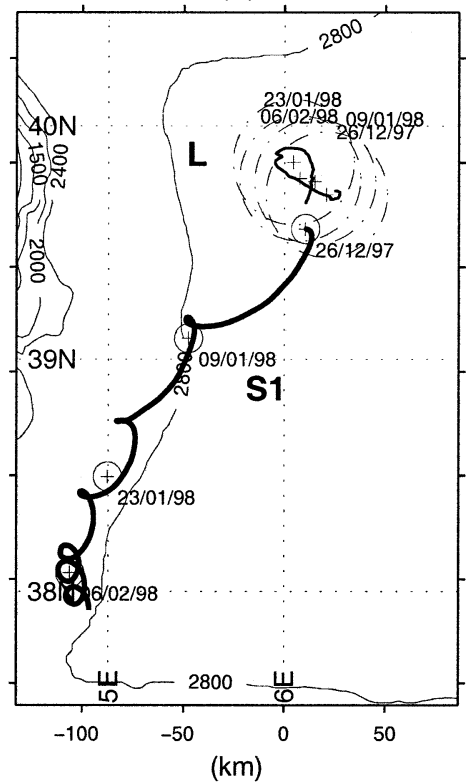

(III)

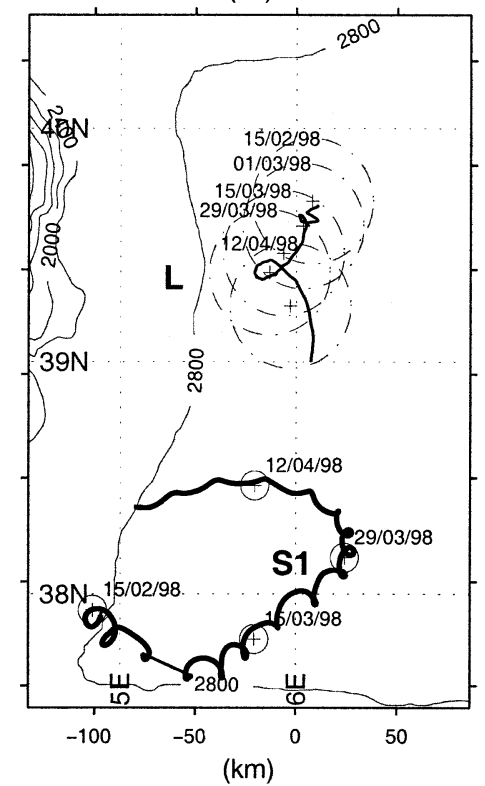

FIG. 6. Trajectories of floats 39 (dark solid) and 14 (light dashed): (I) from 1 Aug 1997 to 26 Dec 1997 , (II) from 26 Dec 1997 to 14 Feb 1998, and (III) from 15 Feb 1998 to 20 Apr 1998. Floats 39 and 14 are first trapped in a mesoscale LIW eddy (L) detaching from the Sardinian vein (I). Then, float 14 remained trapped in L and float 39 began to drift in the small vortex S1 (II), (III). Circle locations are based on the estimates of eddy centers of rotation; their radii correspond to eddy radii. Circles are plotted every 2 weeks.

transition into a distinct and smaller anticyclonic vortex (SCV-S1) characterized by much shorter period rotation (about 3-6 days). This configuration indicates an interaction between the LIW eddy and SCV-S1, which is discussed further in section 5. Another small vortex (SCV-S2), revealed by float 97 and similar to SCV-S1, was also observed in this basin. Indeed, the trajectories appeared similar: "cycloidal" shape (Fig. 10), small radius of curvature, and anticyclonic rotational period of a few days. As for SCV-N1 and SCV-N2, these two eddies, SCV-S1 and SCV-S2, presented no surface signature in NOAA AVHRR SST images. Floats 39 and 97 remained trapped inside SCV-S1 and SCV-S2 from December of 1997 to July of 1998 and from August of 1997 to July of 1998 , respectively.

Estimates of the vortex center locations provide the radial distributions of orbital velocities for the two eddies (Fig. 11). The radius of SCV-S1, defined as before $\left(R_{\max }, U_{\max }\right)$ was about $5-6 \mathrm{~km}$. This result is confirmed by trajectories of floats passing nearby at the same depth ( $\sim 14 \mathrm{~km}$ to the vortex center location) but not influenced by the vortex rotations (not shown). Relative vorticities were estimated as approximately $-f / 2.4$ inside the core (very near the center $<3 \mathrm{~km}$ ) and then decreasing with increasing radial distance.

SCV-S2 was sampled by float 97 only very near the center. Orbital velocities were still large, corresponding to a relative vorticity of about $-f / 3$ near the eddy center $(<1 \mathrm{~km})$. The radius of SCV-S2 was also limited to about $5 \mathrm{~km}$, since a float at the same depth passing about
$9 \mathrm{~km}$ from the vortex center location did not show any anticyclonic rotation (Fig. 12).

The relative vorticities of the cores correspond to Rossby numbers of -0.4 for SCV-S 1 and -0.3 for SCV$\mathrm{S} 2$, slightly less than for SCV-N1 and SCV-N2. For both SCV-S1 and SCV-S2, the eddy characteristic length scale was much smaller than the internal radius of deformation ( $\sim 15-20 \mathrm{~km}$ in the Algerian Basin), and the eddy lifetime was on the order of $1 \mathrm{yr}$ (at least 7 months for SCV-S1 and 10 months for SCV-S2). These observations confirm that these eddies can again be identified as submesoscale coherent vortices as were SCV-N1 and SCV-N2.

\section{Discussion}

\section{a. $n W M D W$ spreading and the origin of northern $S C V s$}

SCV-N1 and SCV-N2 were revealed at the outset in the mixed patch of the so-called Medoc area just after the violent winter mixing events. In addition, temperature signals, high anticyclonic vorticities, and small radii in comparison with the ambient deformation radius just out of the convection area indicated these SCVs evidently had an origin related to deep convection. Northern SCVs (lifetime exceeding $0.5 \mathrm{yr}$ ) advected nWMDW more than $300 \mathrm{~km}$ away from the Medoc area (section 3). Because of their small radius and despite their long lifetime, SCVs have rarely been observed in the past since all previous measurements were too short 

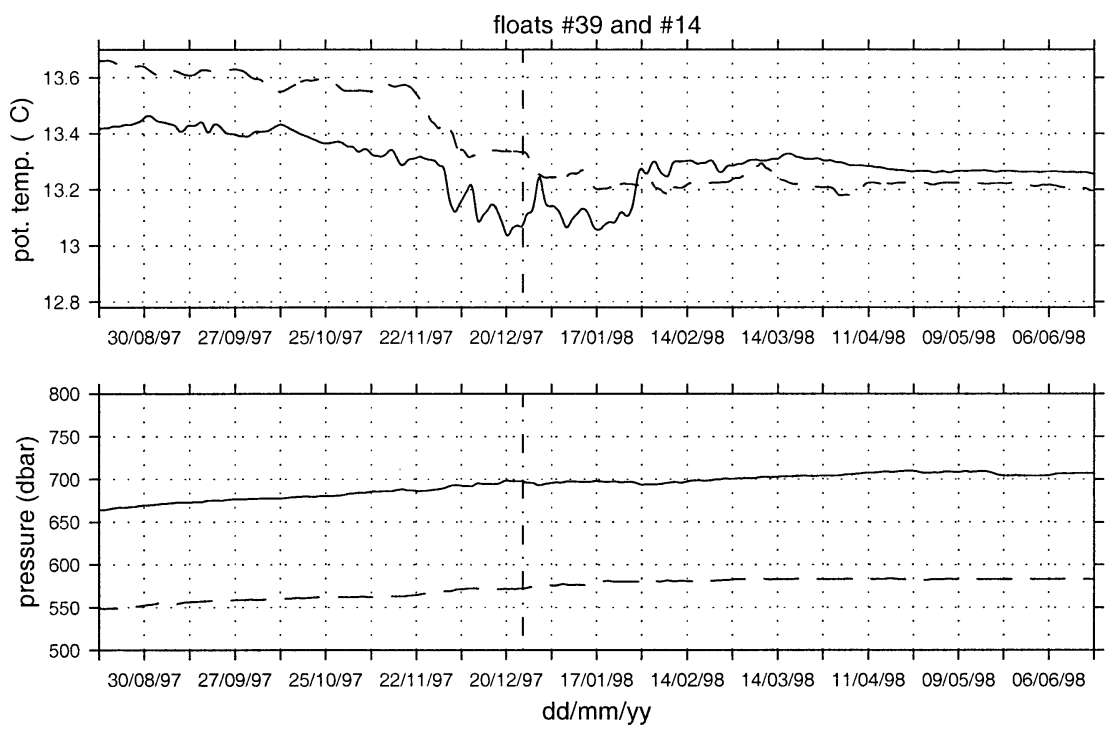

FIG. 7. Time series of potential temperature and pressure measured by float 39 (solid) and float 14 (dashed) corresponding to the situation presented in Fig. 6. Note that float 14 remained in the LIW eddy L whereas float 39 remained in L only until 26 Dec 1997 (vertical dotteddashed line) and then began to drift in S1.

and too coarse. Eddies with radius and rotational period very similar to SCV-N1 and N2 have been observed at convective sites in the Labrador and Mediterranean Seas but only over a few weeks (Gascard 1978; Gascard and Clarke 1983). Thus the long-term behavior of SCVs and their contribution to the large-scale spreading of the newly formed deep waters have not been recognized previously. In the Weddell Sea, Gordon (1978) observed an eddy with large vertical extension and small radius composed of newly formed deep waters, which survived into summer and was the first evidence of longevity for such eddies originating from deep convection.

As already mentioned, Madec et al. (1991) and Send et al. (1996) reported entrainment of nWMDW by the northern boundary current along the continental slope of the Gulf of Lion (the so-called bleeding effect) that could account for $50 \%$ in the export of nWMDW out of the convection area (Send et al. 1996). Our obser-
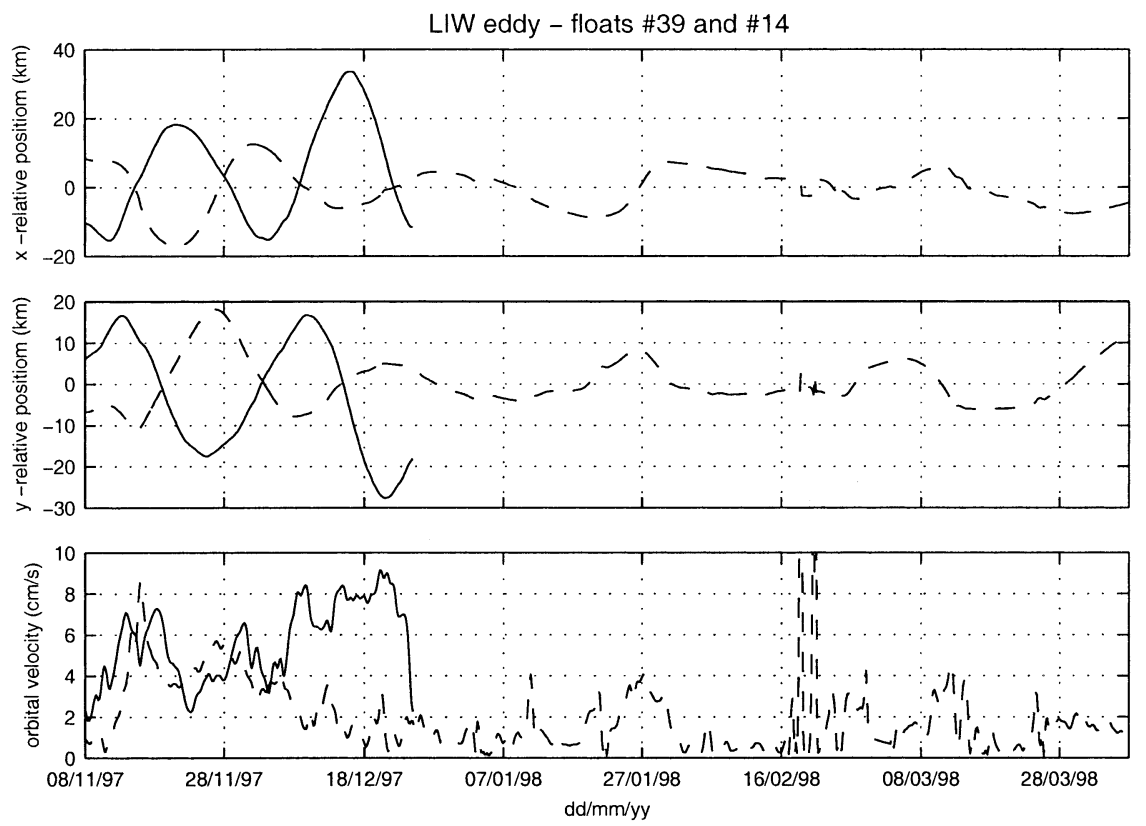

FIG. 8. Time series of orbital velocities and positions of floats 39 (solid) and 14 (dashed) relative to the center of the LIW eddy. 

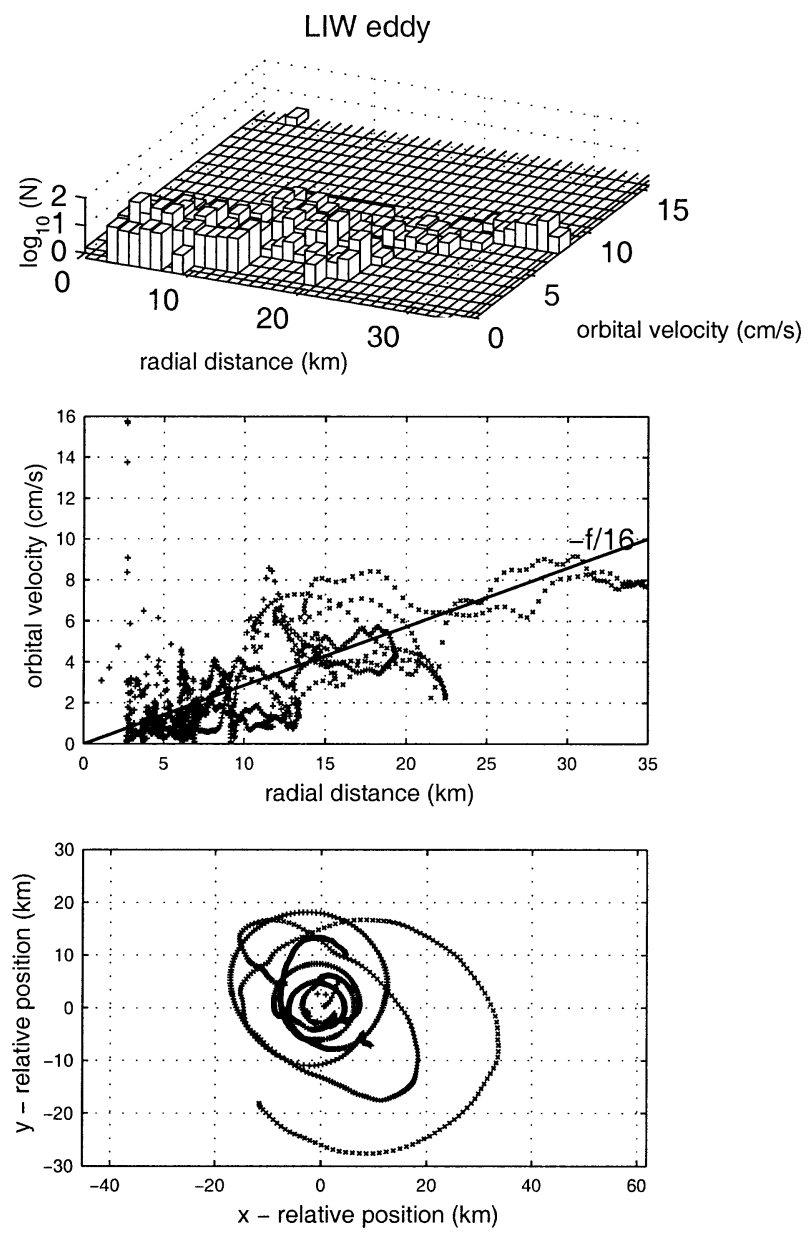

FIG. 9. (bottom) Radial structure of the orbital velocities of the LIW eddy inferred from an estimate of the eddy center of rotation as a function of time. (middle) and (top) Positions of floats $39(\times)$ and $14(+)$ relative to the center of the LIW eddy, where $N$ is the number of measurements in each $2 \mathrm{D}$ class.

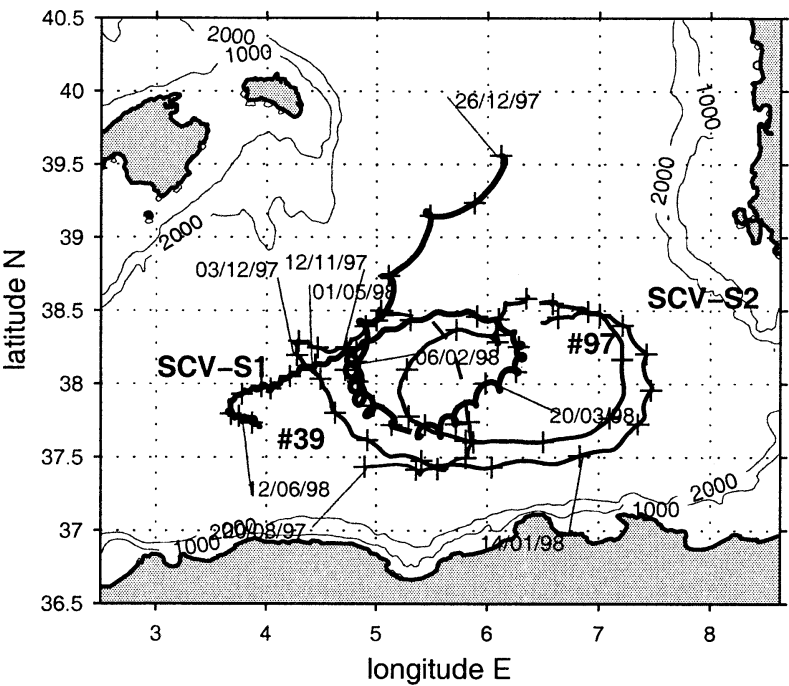

FIG. 10. Trajectories of floats 39 (thick solid) and 97 (thin solid) that reveal SCVs (SCV-S1 and SCV-S2) in the Algerian Basin, and 14 and 39 (thin) that reveal a LIW eddy from the 1997-98 MATER/ MAST3 experiment.

vations indicate a very different process by which nWMDW can spread far away from the formation area. What would be the relative importance of each process? The total amount of nWMDW formed in 1994-95 can be very roughly approximated by the volume of the mixed patch (west-east extension $\sim 100 \mathrm{~km}$; northsouth extension $\sim 50 \mathrm{~km}$; vertical scale $\sim 2000 \mathrm{~m}$ ) in the Medoc area: $V_{\mathrm{mp}} \simeq 10^{4} \mathrm{~km}^{3}$. A single SCV represents a volume of about $V_{\mathrm{scv}} \simeq 10^{2} \mathrm{~km}^{3}$. This is $1 \%$ of new water production. Two floats revealing the existence of two SCVs in February 1995 were situated in a sector corresponding to approximately $1 / 5$ of the total surface area of the mixed patch. We may have missed many other similar features. Killworth (1979) argued that a large number of chimneys (17) could pepper the mixed patch without ever being observed, based on statistical arguments and scale considerations. In the same way, the number of SCVs generated each year could be

TABLE 1. Descriptive information for the SCVs and the LIW eddy. Here $T_{r}$ is the range of rotational periods, $\zeta$ is the relative vorticity, $R_{\max }$ is the estimated radius, $U_{\max }$ is the orbital velocity at this radius, $H$ is the half thickness, Ro is an estimation of Rossby number, Bu is an estimation of Burger number, $T_{\mathrm{obs}}$ is the lifetime observed, and $V_{\text {drift }}$ is the advection speed; $P_{\text {float }}$ and $\theta_{\text {float }}$ are the pressures and potential temperatures measured by floats.

\begin{tabular}{|c|c|c|c|c|c|}
\hline & SCV-N1 & SCV-N2 & SCV-S1 & SCV-S2 & LIW eddy \\
\hline$T_{r}$ (days) & $2.6-4.0$ & $3.7-5.5$ & $3.0-6.0$ & $4.3-6.0$ & $19.6-27.4$ \\
\hline$\zeta\left(\mathrm{s}^{-1}\right)$ & $-f / 2$ & $-f / 2.4$ & $-f / 2.4$ & $-f / 3$ & $-f / 16$ \\
\hline$U_{\max }\left(\mathrm{m} \mathrm{s}^{-1}\right)$ & & 9 & 9 & & 9 \\
\hline$R_{\max }(\mathrm{km})$ & $5(<10)$ & $5(\geq 5)(<10)$ & $5(\geq 5)(<14)$ & $5(\geq 2)(<9)$ & $30(\geq 25)(<40)$ \\
\hline$H(\mathrm{~km})$ & $\geq 0.6$ & & & & \\
\hline $\operatorname{Ro}(\zeta / f)$ & -0.5 & -0.4 & -0.4 & -0.3 & -0.06 \\
\hline $\mathrm{Bu}^{1 / 2}$ & 0.24 & & & & \\
\hline$T_{\mathrm{obs}}$ (months) & $>5$ & $>5$ & $>7$ & $>10$ & $>5$ \\
\hline$V_{\text {drift }}\left(\mathrm{cm} \mathrm{s}^{-1}\right)$ & $5.3 \pm 2.7$ & $5.5 \pm 2.4$ & $4.2 \pm 2.3$ & $4.8 \pm 2.4$ & $2.1 \pm 1.8$ \\
\hline$P_{\text {float }}(\mathrm{dbar})$ & 1400 & 450 & 700 & 600 & $600 / 700$ \\
\hline$\theta_{\text {float }}\left({ }^{\circ} \mathrm{C}\right)$ & $12.89 \pm 0.00$ & $13.01 \pm 0.06$ & $13.24 \pm 0.12$ & $13.24 \pm 0.06$ & $13.40 \pm 0.18 / 13.29 \pm 0.11$ \\
\hline
\end{tabular}



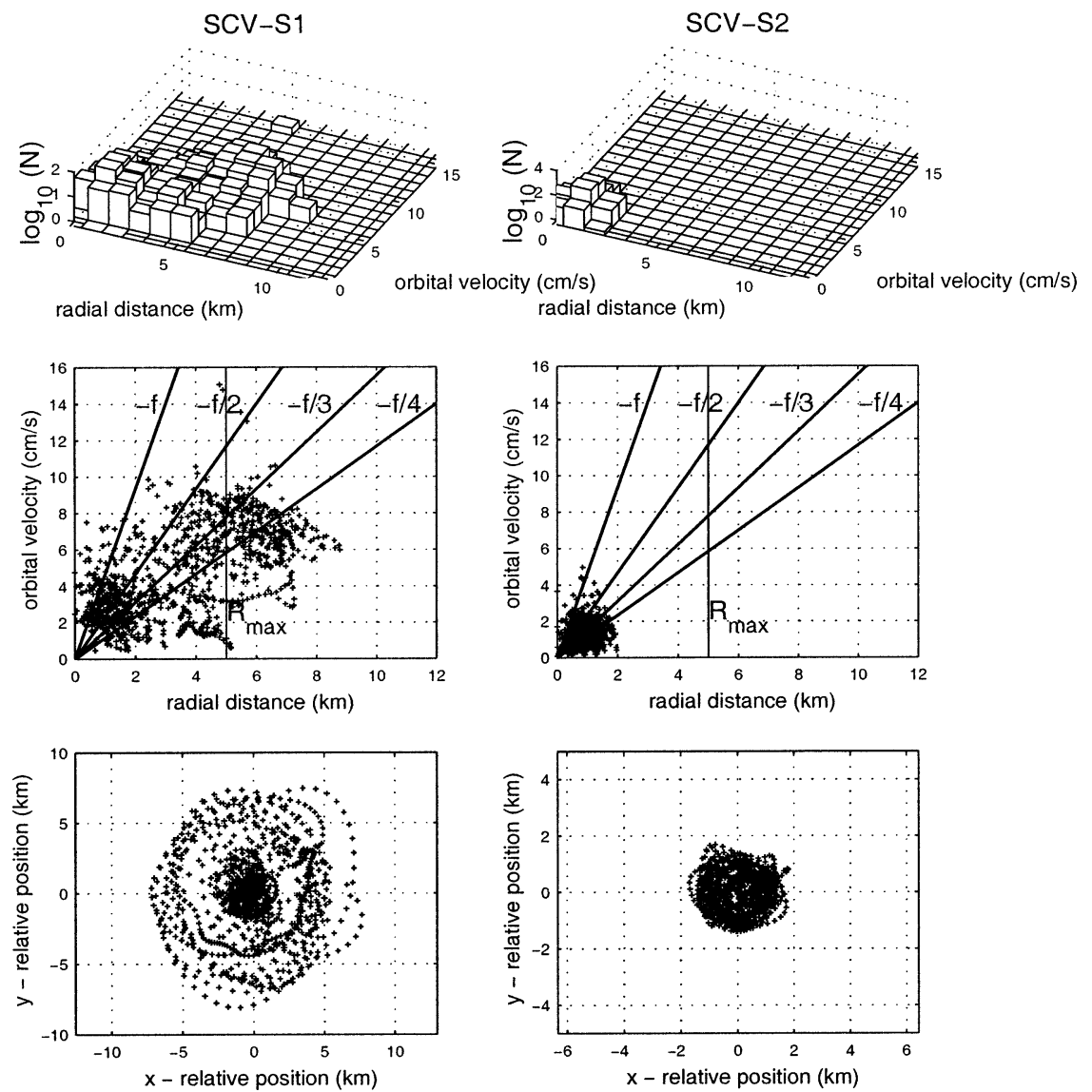

FIG. 11. (bottom) Positions of floats relative to the centers of the eddies. (middle) Radial structure of the orbital velocities of SCV-S1 and SCV-S2 inferred from an estimate of the eddy center of rotation as a function of time. (top) Two-dimensional histograms of the number of measures, where $N$ is the number of measurements in each 2D class.

on the order of 10-20 and would represent $10 \%-20 \%$ of the total amount of nWMDW formed in a particular year. In fact, restricting our estimates to the deep-convection part of the ventilation (deeper than $1 \mathrm{~km}$ ), SCVs could account for as much as $20 \%-40 \%$ of the ventilation of the deep western Mediterranean Basin.

\section{b. Origin of southern SCVs}

The northern SCVs (SCV-N1 and SCV-N2) clearly have a deep convective origin. What about the origin of the southern SCVs? We present four arguments to demonstrate that the SCVs observed in the northern and southern basins of the western Mediterranean Sea have the same origin.

The first argument is as follows. Northern SCVs are clearly capable of migrating from the northern to the southern basin of the western Mediterranean Sea. SCVN2 was still robust when it entered the Algerian Basin in July of 1995 as indicated by float 07 just before it was recovered. The last position of float 07 was near $39^{\circ} 25^{\prime} \mathrm{N}, 6^{\circ} 45^{\prime} \mathrm{E}$, which is 10 miles away from the first observation of SCV-S1 $\left(39^{\circ} 30^{\prime} \mathrm{N}, 6^{\circ} 20^{\prime} \mathrm{E}\right)$ at float 39 in
1997 (Fig. 13). This does not mean that SCV-N2 and SCV-S1 are the very same eddy, especially because there are 2 years between them, but it does indicate that northern SCVs are observed at the same locations as southern SCVs.

The second argument goes thus. Southern SCV-S1 and SCV-S2 are similar to the northern SCV-N1 and SCV-N2 in scales and dynamical characteristics (Table 1: radius of $5 \mathrm{~km}$, rotational period of a few days, strong relative anticyclonic vorticity of about $-f / 3$ to $-f / 2$, and lifetime of several months). Also, the radii of SCV$\mathrm{S} 1$ and SCV-S2 are significantly smaller than the ambient deformation radius (as for SCV-N1 and SCV-N2 radii just out of the convection area). Their high Rossby numbers and strong potential vorticity anomalies indicate a homogeneous core originating from an especially weakly stratified region such as the Medoc area.

A third argument is based on high-frequency temperature signals obtained at the time the floats were trapped in the LIW eddy and revealing an interaction with a cold-core eddy: a northern SCV. Figure 14 shows the relative positions of floats 39 and 14 trapped initially in the LIW eddy together with orbital velocities and 


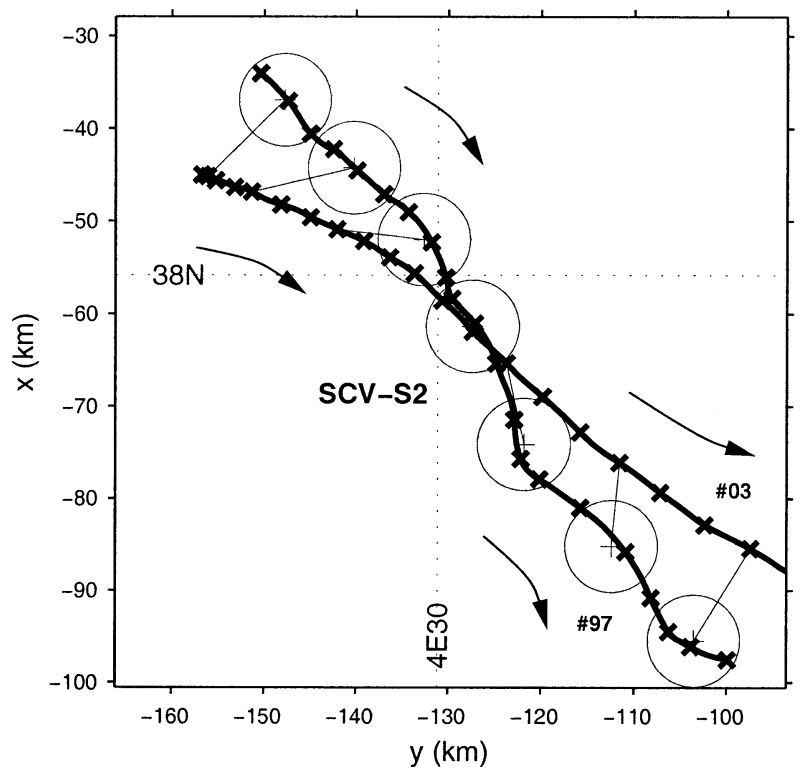

FIG. 12. Trajectories of floats 03 (situated at around 600-m depth) and float 97 (situated at around 600-m depth) between 3 and $23 \mathrm{Dec}$ 1997. A cross marks each day. Every 3 days, a thin circle with 5$\mathrm{km}$ radius centered on SCV-N2 center positions and a thin line between the center and float 03 position are plotted. The minimum distance between the center of SCV-S2 and float 03, which is not influenced by anticyclonic rotation, is about $9 \mathrm{~km}$.

high-resolution temperature samples (unfiltered, sampled every $4 \mathrm{~h}$ ) until float 93 left the LIW eddy to drift in SCV-S1 on 26 December 1997. Initially, from 13 November 1997 to 3 December 1997, the two floats were located at about the same distance from the center of the LIW eddy and were almost diametrically opposed. During that period, the LIW eddy bulk temperature decreased by $0.25^{\circ} \mathrm{C}$ at both 550 and $700 \mathrm{~m}$, under the influence of a relatively cold environment. The highfrequency variability can be interpreted as strong interactions between the warm LIW eddy and the cold environment, in particular at the periphery of the LIW eddy. Around 8 December 1997 (Fig. 6), float 39 moved farther away from the center and simultaneously the high-frequency temperature variability rose to up to $\pm 0.1^{\circ} \mathrm{C}$ (Fig. 14). Float 14 , nearer to the eddy center, had temperatures at a constant value of $13.32^{\circ} \mathrm{C}$. Thus float 39 , located at the edge of the LIW eddy, apparently shows greater interactions between the warm LIW eddy and the cold environment.

Temperatures at float 39 at 700-m depth reached as low as $12.95^{\circ}-12.97^{\circ} \mathrm{C}$ during $16 \mathrm{~h}$ on 21 December 1997 , which is $0.4^{\circ} \mathrm{C}$ colder than temperatures reported by float 14 at the same time. Such a cold anomaly, at this depth, could only correspond to nWMDW, the coldest water found in the western Mediterranean Sea at this particular depth. SCVs such as SCV-N1 or SCV-N2 that originate from the Medoc area are features able to propagate such cold anomalies at 700-m depth in the Algerian Basin. Strikingly, a few days later, on 26 De-

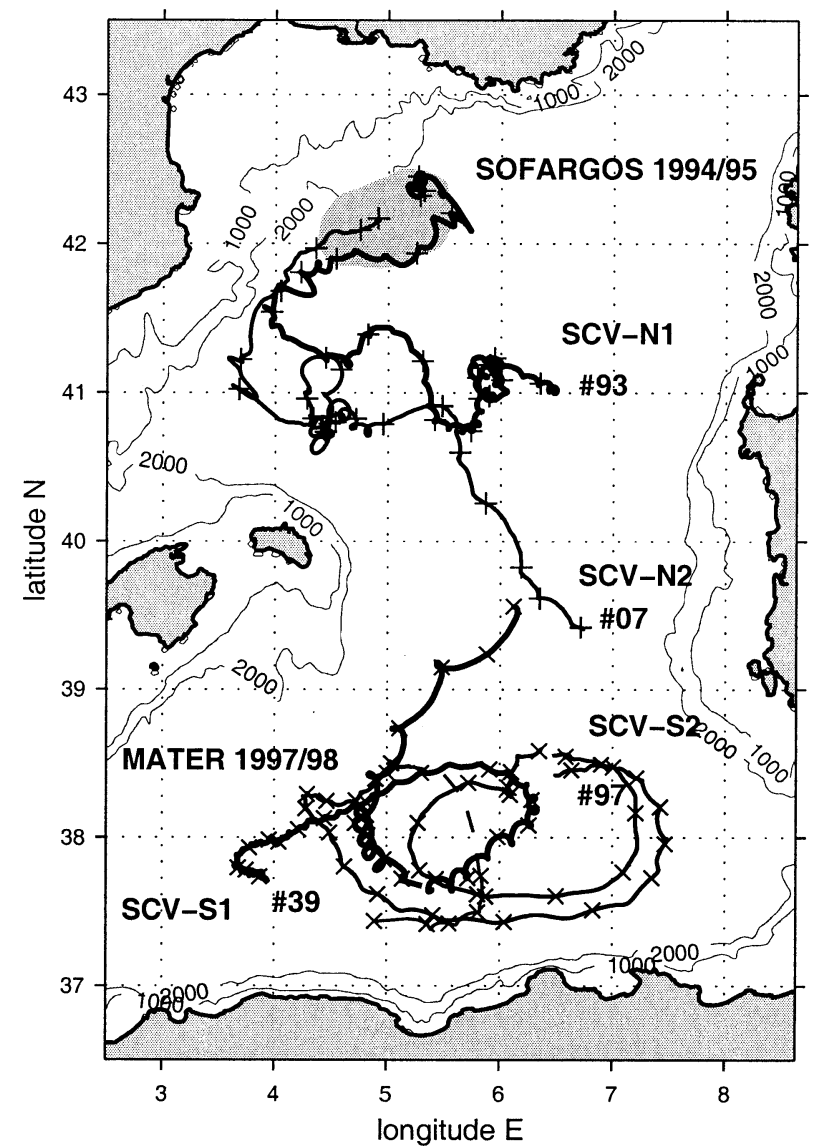

FIG. 13. Trajectories of floats 93 and 07 ( + on dark solid lines) from SOFARGOS/MAST2 1994-95 experiment and trajectories of floats 39 and 97 ( $\times$ on dark solid lines) from MATER/MAST3 199798 experiment, revealing SCVs in the Algero-Provençal Basin.

cember 1997, strong interactions between the LIW eddy and an SCV (SCV-S1) appeared clearly from the trajectories of floats 39 and 14 (section $4 \mathrm{~b}$ and Fig. 6). Since float 39 was initially trapped in the LIW eddy core, escaped from the LIW, and was then trapped in an SCV-like feature at about the same time as reporting cold temperature anomalies, we conclude that an SCV originating from the Medoc area and leaving the northern basin interacted with an LIW eddy a soon as it entered in the Algerian Basin.

The fourth argument is that a simple diffusive model based on the float's temperatures and on a climatology of the region supports the hypothesis of a northern origin for the southern SCVs. We constructed a climatological dataset of the potential temperature of the western Mediterranean Sea for the past $6 \mathrm{yr}$ with a horizontal spacing of $10 \mathrm{~km}$ at 53 levels using about 400 CTD stations from this area from July of 1994 to April of 2000. This dataset is used to simulate the temperature evolution of a fluid parcel $\left(d T / d t=\kappa \nabla^{2} T\right.$, where $\kappa$ is diffusion coefficient), characterized by an initial potential temperature of $12.9^{\circ} \mathrm{C}$ and a volume similar to an SCV $(\sim 5-$ $\mathrm{km}$ radius, $1000-1500-\mathrm{m}$ height) and advected from the 

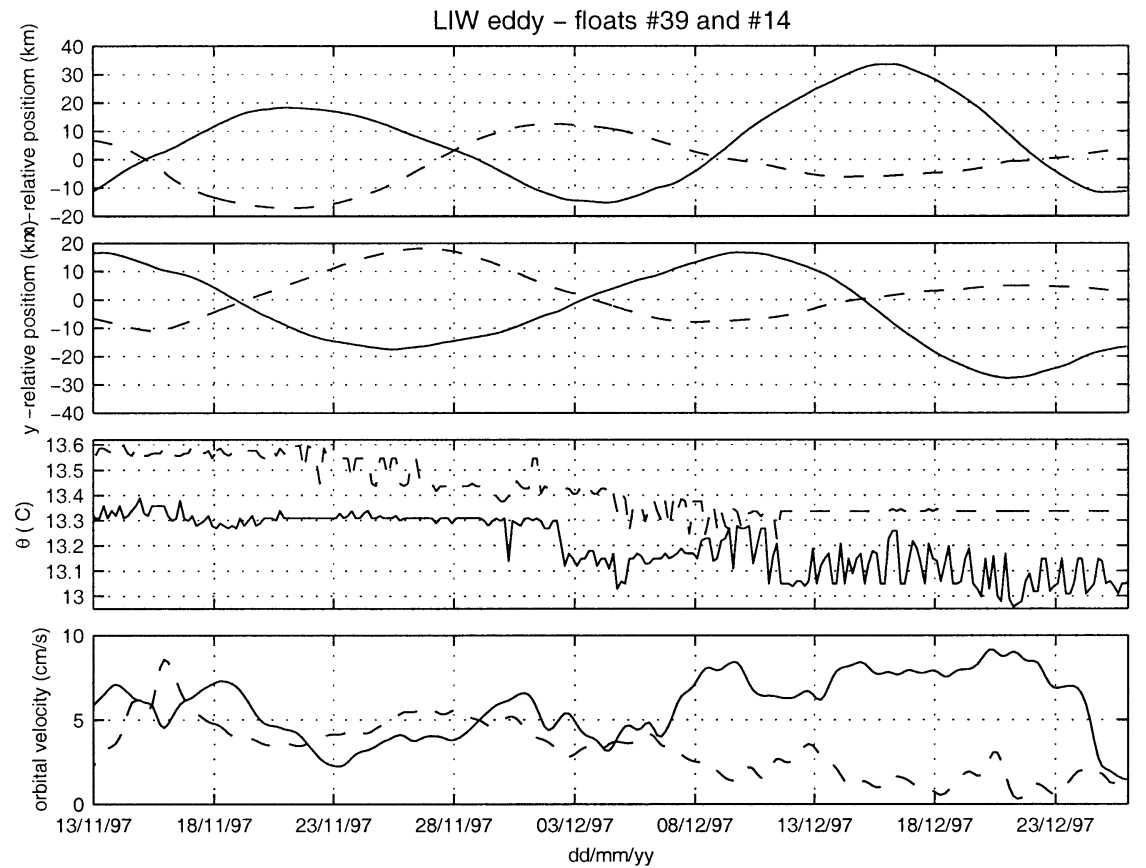

FIG. 14. Time series of potential temperatures, orbital velocities, and positions of floats 39 (solid) and 14 (dashed) relative to the center of the LIW eddy.

Medoc area in the north to the Algerian Basin in the south, according to actual SCV trajectories and speeds. With a horizontal diffusion coefficient of $O(1) \mathrm{m}^{2} \mathrm{~s}^{-1}$ and a vertical diffusion coefficient of $O\left(10^{-4}\right) \mathrm{m}^{2} \mathrm{~s}^{-1}$ (e.g., Hebert et al. 1990), the simple model shows that the temperature anomalies typical of a northern SCV at the beginning of its life would gradually disappear in the upper and lower parts of a SCV on a timescale of approximately 150 days.

When it started drifting in SCV-S1, float 39 measured potential temperatures of about $13.05^{\circ} \mathrm{C}$. This suggests nWMDW at this depth in this region, although a little bit warm. Temperatures then increased up to $13.3^{\circ} \mathrm{C}$ in July of 1998 (Fig. 15), and any direct link to deep convection based only on temperature faded away. Accordingly, temperatures at the later float 97 (SCV-S2) do not indicate a deep convection origin.

The long-term evolution of SCV potential temperature vertical structure can be described as follows: initially, the temperature inside of the SCV core, made of pure nWMDW, must be about $12.9^{\circ} \mathrm{C}$ from top to bottom. As time progresses, the upper part of the core warms under the influence of the warm and salty LIW $\left(>13.3^{\circ} \mathrm{C}\right.$ ) surrounding it (see float 07, sampling SCV$\mathrm{N} 2$; section $3 \mathrm{~b}$ ) and the lower part is cooled by WMDW $\left(\sim 12.8^{\circ} \mathrm{C}\right)$. So, one should not expect to measure temperatures in the Algerian Basin of about $12.9^{\circ} \mathrm{C}$ typical of nWMDW at 700-m depth in SCVs that would come from the Medoc area. Floats 39 and 97 reported temperatures that are consequently not in contradiction with a northern origin several months or a year before, be- cause these floats would have been trapped in the upper part of SCVs coming from the north.

Thus, based on these arguments, we conclude that the most credible formation process for SCV-S1 and SCV$\mathrm{S} 2$ is deep convection. The SCVs would have been formed during the previous winter season in the Medoc area and were discovered several months later, when they entered the Algerian Basin during the MATER 1997-98 experiment. This conclusion highlights the importance of the spreading of nWMDW by SCVs and their implications for the thermohaline circulation of the western Mediterranean Sea.

McWilliams (1985) concluded that SCVs are most likely generated by diapycnal mixing followed by cyclostrophic adjustment. Because the most frequently invoked source of mixing is surface forcing, especially in convectively forced regions, SCVs are supposed to be mostly formed by wintertime intermediate convection and deep convection.

SCVs can propagate very far from the source region because of their long lifetimes. Similar to what McWilliams (1985) hypothesized for SCVs observed in the North Atlantic and coming from the Labrador Sea, an origin linked to deep convection in the Medoc area is a plausible scenario for the SCVs that we discovered in the Algerian Basin.

Based on rotating-tank experiments, Hedstrom and Armi (1988) predict that SCV anticyclonic vorticity and height should decrease with time. They also suggest that the radius of these eddies will remain constant until a final decay by the shedding effect. However, we were 

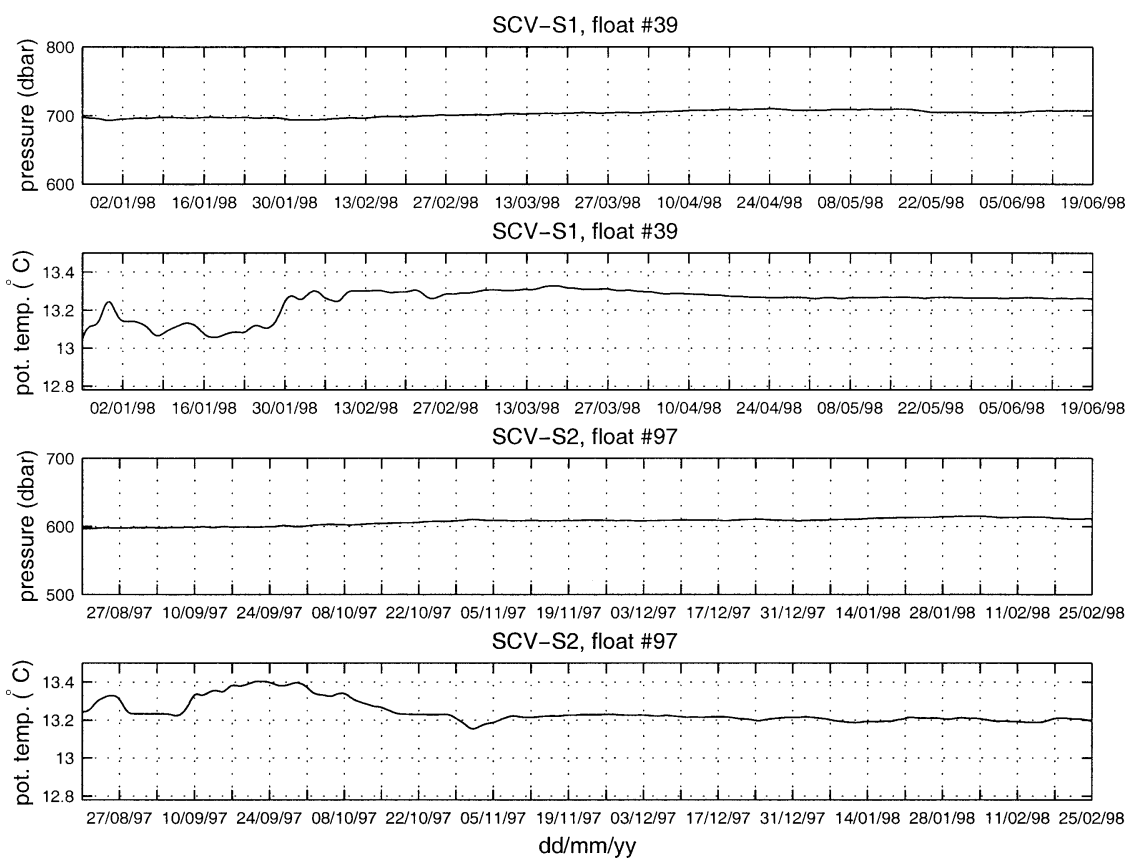

FIG. 15. Time series of potential temperature and pressure measured by float 39 and float 97 drifting in SCV-S1 and SCV-S2, respectively.

unable to detect any trend in vorticity, height, or radius in the observed SCVs, because of lack of horizontal/ vertical resolution.

The small size of SCVs and their temperature characteristics (very difficult to dissociate from the background field) make them very difficult to observe with basic measurements such as CTD casts. Only Lagrangian experiments have the potential to show the coherent character of SCVs with sufficient time resolution and duration. SOFAR Lagrangian techniques are adaptable for a dedicated study following SCVs in near-real time over a long period of time since SOFAR floats emit acoustic signals instead of receiving them as RAFOS ones do.

\section{Conclusions}

Our observations in the Mediterranean Sea document a new aspect of the large-scale circulation of the newlyformed deep waters in the western Mediterranean Sea. The SOFARGOS 1994-95 and MATER 1997-98 Lagrangian experiments revealed the existence of submesoscale coherent vortices in the northern and southern parts of the western Mediterranean Sea, respectively. The SCVs are subsurface eddies, characterized by a radius of about $5 \mathrm{~km}$, a relative vorticity reaching $-f / 2$, and a large aspect ratio of greater than 0.1. Their lifetimes may exceed $1 \mathrm{yr}$. The origin of northern SCVs is clearly related to deep convection in the Gulf of Lion. The SCVs discovered in the Algerian Basin could also have originated from deep convection in the Gulf of Lion. Consequently, SCVs can advect newly formed western Mediterranean deep waters several hundreds of kilometers from the source region, crossing the western Mediterranean Sea. Based on SOFARGOS observations, SCVs can propagate $300 \mathrm{~km}$ away from the source region, over a period of 5 months. Based on MATER, SCVs can propagate $500 \mathrm{~km}$ and over $1.5 \mathrm{yr}$ if we assume that SCVs discovered in the Algerian Basin were generated in the Medoc area.

During their long journey, SCVs slowly transfer characteristics of nWMDW to the ambient waters. The upper part of the nWMDW SCVs is warmed by LIW, and the lower part is cooled by WMDW. In particular, this involves eddy-eddy interactions; the MATER experiment suggested that an SCV composed of nWMDW strongly interacted with an LIW eddy. This interaction might especially influence the large-scale spreading of intermediate and deep waters in the western Mediterranean Sea. The LIW eddy is also a newly discovered feature and is the main topic of another paper just submitted for publication.

These Lagrangian experiments offer new insight into the large-scale spreading of nWMDW (following deep convection) and, consequently, thermohaline circulation in the Mediterranean Sea. SCVs must be included in understanding the circulation of deep waters in the western Mediterranean Sea. Further studies about the formation mechanisms and behaviors are still needed to quantify the implications of these eddies.

Acknowledgments. The SOFARGOS and MATER experiments were funded by the European Union as part of the MAST2 and MAST3 programs, under Contracts 
MAS2-CT92-0046 and MAS3-CT96-0051. We acknowledge Antonio Lourenço and Catherine Rouault for their expertise in operating floats and in data processing. The comments and suggestions from anonymous reviewers were appreciated and helped to improve this manuscript significantly from an earlier version. Author P. Testor is supported by a DGA grant.

\section{REFERENCES}

Gascard, J.-C., 1978: Mediterranean deep water formation, baroclinic eddies and ocean eddies. Oceanol. Acta, 1, 313-315.

— and A. C. Clarke, 1983: The formation of Labrador Sea water Part II: Mesoscale and smaller-scale processes. J. Phys. Ocean$o g r .$, 13, 1779-1797.

Gordon, A. L., 1978: Deep Antarctic convection west of Maud Rise. J. Phys. Oceanogr., 8, 600-612.

Hebert, D., N. Oakey, and B. Ruddick, 1990: Evolution of a Mediterranean salt lens: Scalar properties. J. Phys. Oceanogr., 20, $1468-1483$.

Hedstrom, L., and A. Armi, 1988: An experimental study of homogeneous lenses in a stratified fluid: Scalar properties. J. Fluid Mech., 191, 535-556.

Jones, H., and J. Marshall, 1997: Restratification after deep convection. J. Phys. Oceanogr., 27, 2276-2287.

Killworth, P. D., 1979: On "chimney" formation in the ocean. J. Phys. Oceanogr., 9, 531-554.
1983: Deep convection in the World Ocean. Rev. Geophys., 21 (1), 1-26

Legg, S., and J. Marshall, 1993: A heton model of the spreading phase of open-ocean deep convection. J. Phys. Oceanogr., 23, 1040-1056.

—, H. Jones, and M. Visbeck, 1996: A heton perspective on baroclinic eddy transfer in localized ocean convection. J. Phys. Oceanogr., 26, 2251-2266.

—_, J. McWilliams, and J. Gao, 1998: Localization of deep convection by a mesoscale eddy. J. Phys. Oceanogr., 28, 944-970.

Madec, G., F. Lott, P. Delecluse, and M. Crepon, 1991: A threedimensional numerical study of deep water formation in the northwestern Mediterranean Sea. J. Phys. Oceanogr., 21, 13491371.

Marshall, J., and F. Schott, 1999: Open-ocean convection: Observations, theory and models. Rev. Geophys., 37, 1-64.

MAST2, 1996: Final scientific report. Contract MAS2-CT92-0046. 1999: Final scientific report. Contract MAS3-CT96-0051.

Maxworthy, T., and S. Narimousa, 1994: Unsteady, turbulent convection into a homogeneous, rotating fluid with oceanographic application. J. Phys. Oceanogr., 24, 865-887.

McWilliams, J., 1985: Submesoscale coherent vortices in the ocean. Rev. Geophys., 23 (2), 165-182.

MEDOC Group, 1970: Observation of formation of deep water in the Mediterranean Sea, 1969. Nature, 227, 1037-1040.

Millot, C., 1999: Circulation in the western Mediterranean Sea. J. Mar. Sys., 20, 423-442.

Send, U., J. Font, and C. Mertens, 1996: Recent observation indicates convection's role in deep water circulation. Eos, Trans. Amer. Geophys. Union, 77 (7), 61-65. 